\section{Análise da contribuição das variáveis meteorológicas no estresse térmico associada à morte de cortadores de cana-de-açúcar}

\author{
Analysis of the contribution of meteorological \\ variables to thermal stress associated with \\ death among sugarcane cutters
}

\author{
1 Fundação Jorge Duprat \\ Figueiredo de Segurança e \\ Medicina do Trabalho, Centro \\ Estadual de Santa Catarina, \\ Florianópolis, Brasil. \\ 2 Fundação Jorge Duprat \\ Figueiredo de Segurança \\ e Medicina do Trabalho, \\ Centro Técnico Nacional, \\ Campinas, Brasil. \\ Correspondência \\ D. P. Bitencourt \\ Fundação Jorge Duprat \\ Figueiredo de Segurança e \\ Medicina do Trabalho, Centro \\ Estadual de Santa Catarina. \\ Rua Silva Jardim 213, \\ Florianópolis, SC 88020-200, \\ Brasil. \\ daniel@fundacentro.sc.gov.br
}

\section{Abstract}

Sugarcane cutters perform heavy work under adverse conditions. Environmental factors can be an aggravating factor for thermal stress among these workers. This study analyzed the atmospheric conditions on the day of death of 14 sugarcane cutters in São Paulo State, Brazil. Historical data for temperature, humidity, wind, and sunlight were compared with the same meteorological variables on the days the deaths that occurred. The meteorological data were obtained from a reanalysis of the National Center for Environmental Prediction/National Center for Atmospheric Research (NCEP/NCAR). In 10 of the 14 cases, the temperature on the day of death was higher than or equal to the mean plus the standard deviation. In six of these cases, the temperature reached or came close to record levels. These findings suggest that atmospheric conditions can be an important factor in the analysis of overall work conditions for sugarcane cutters.

Heat Stress Disorders; Occupational Health; Atmosphere
Daniel Pires Bitencourt 1

Álvaro César Ruas 2

Paulo Alves Maia 2

\section{Introdução}

A área utilizada para o plantio de cana-de-açúcar no Brasil em 2008 foi de 8.141.228ha, com uma produção de 648.973.981 toneladas (Instituto Brasileiro de Geografia e Estatística. Levantamento sistemático da produção agrícola. http:// www.ibge.gov.br/home/estatistica/indicadores/ agropecuaria/lspa/default.shtm, acessado em 08/Set/2009). No ano de 2009 a área plantada no Brasil aumentou em 3,2\%, possivelmente devido à procura de alternativas ao uso do petróleo.

No Estado de São Paulo, desde meados da década de 80, tem aumentado a mecanização do corte da cana devido aos problemas ambientais gerados pela queima e os sérios problemas de saúde característicos da realização manual desta tarefa 1 . Segundo Petti \& Fredo 2 , atualmente 40,7\% das áreas cultivadas em São Paulo já são exploradas por meio de máquinas agrícolas. Entretanto, mesmo que a tendência de aumento da mecanização continue, há áreas que exigirão muitos investimentos na adequação do terreno para que possam ser exploradas por esse processo. No Brasil, há atualmente 335 mil cortadores de cana-de-açúcar 3.

Embora as tecnologias aplicadas na produção da cana-de-açúcar tenham evoluído nos últimos anos, as condições de trabalho oferecidas aos trabalhadores envolvidos no processo continuam extremamente precárias. O empre- 
go formal tem crescido no Estado de São Paulo, mas em índices muito aquém do aumento da produção.

A poluição provocada pelo processo de queima da cana-de-açúcar causa impactos ambientais 4 e problemas respiratórios para os trabalhadores e para a população ao redor do cultivo 5,6.

A precariedade do trabalho nos canaviais é pouco abordada no meio científico, mas alguns autores relataram as más condições de trabalho, as quais se submetem os cortadores de cana no atual modelo de produção da agroindústria canavieira 1,7,8,9,10. Alessi \& Navarro 7 destacam a extensa jornada de trabalho, a intensificação do ritmo de trabalho, o pagamento por produção, o decréscimo real do valor dos salários e o descumprimento dos direitos trabalhistas como fatores marcantes nas características gerais do trabalho no campo. Os autores advertem que esses fatores afetam muito a saúde dos trabalhadores rurais. Além disso, os trabalhadores do corte da canade-açúcar, na maioria das vezes, alimentam-se mal, são transportados de forma inadequada, bebem pouco líquido durante a jornada de trabalho e descansam apenas poucos minutos após alimentarem-se. Os trabalhadores não dispõem de todos os equipamentos de proteção individual (EPI) necessários e frequentemente improvisam para evitar danos físicos. O local do cultivo geralmente não possui equipamentos de primeiros socorros, condições mínimas de higiene e instalações sanitárias.

Além de toda a precariedade nas condições de trabalho, o cortador de cana enfrenta, diariamente, uma carga de trabalho excessivamente pesada. Segundo Juttel 3, que realizou uma pesquisa ergonômica sobre o corte manual de canade-açúcar no interior de São Paulo, durante uma jornada de trabalho o cortador de cana alcança a impressionante marca de 3.792 golpes de facão, 3.994 flexões de coluna e 11,5 toneladas de cana cortadas.

As trocas térmicas com o ambiente, necessárias ao equilíbrio térmico do corpo, são dificultadas por causa das vestimentas que cobrem todo o corpo. A atividade física e as condições ambientais favorecem a produção interna de calor, resultando em sobrecarga térmica. Esse processo pode ser traduzido em solicitações fisiológicas capazes de causar as chamadas doenças do calor. As consequências fisiológicas da sobrecarga térmica dependem da carga de calor e das características individuais do trabalhador exposto, podendo resultar em irritabilidade inexplicável, confusão mental, câimbras, fadiga severa repentina e outras disfunções com potencial de levar à morte no caso de falência do sistema termorregulador do corpo.
O efeito do calor sobre a saúde de trabalhadores, focando uma atividade específica executada em ambientes externos, é pouco explorado cientificamente. Entretanto, há vários estudos que relacionam o calor do ambiente e a saúde das pessoas, mostrando que há aumento dos índices de mortalidade da população durante ondas de calor 11. Muitos artigos recentes discutem sobre esse tema utilizando dados de mortalidade durante a ocorrência de ondas de calor em várias regiões do mundo 12,13,14,15,16,17. Dhainaut et al. 18 estudaram os excessos de atendimentos e as consequências nas emergências dos hospitais de Paris (França) durante uma onda de calor em 2003. Milojevic et al. 19 encontraram que o efeito da ilha de calor produzida pela área urbana de Londres (Inglaterra) aumenta ainda mais os índices de mortalidade relacionada ao calor. Alguns países, como os Estados Unidos, Coreia e França possuem sistema de alerta para ondas de calor com intuito de minimizar os efeitos negativos à saúde pública 13,14,16.

Entre 2004 e 2008, o serviço Pastoral do Migrante de Guariba (São Paulo) contabilizou 14 óbitos em canaviais do Estado de São Paulo. Alves 8 atribui o ganho por produção como causa primária desses óbitos. $\mathrm{O}$ autor constatou que o alto dispêndio de energia e o esforço excessivo para o corte da cana podem provocar a morte dos trabalhadores ou a perda precoce da capacidade de trabalho. Entretanto, há ainda uma suposição de que o calor frequentemente presente no interior de São Paulo seja também um fator contribuinte para a causa dos óbitos. O risco físico “calor" é mencionado em alguns artigos 1,7,8, mas sem qualquer detalhamento ou quantificação das condições atmosféricas a que os trabalhadores da cana-de-açúcar foram submetidos.

O objetivo deste estudo é trazer para o debate a possível contribuição do clima para a ocorrência de óbito dos cortadores de cana, por meio da comparação das variáveis atmosféricas nos dias em que esses trabalhadores morreram com as da série histórica de 1957 a 2008.

\section{Dados e métodos}

As informações sobre os óbitos de trabalhadores em atividades a céu aberto no corte de cana-deaçúcar foram obtidas junto a Pastoral do Migrante de Guariba 20. Os óbitos ocorreram no próprio canavial, no caminho para o hospital ou após a internação hospitalar. Neste estudo, utilizam-se 14 casos de óbitos ocorridos entre outubro de 2005 e maio de 2008. Apesar do esforço dessa organização em fazer esses registros, é possível que mais mortes tenham ocorrido, tanto durante es- 
se período como no anterior a outubro de 2005. Há também casos registrados que não possuem informação de data precisa e, portanto, não puderam ser utilizados neste estudo. Uma falha importante nos dados diz respeito à ausência de registro para a causa da maioria das mortes. Os locais que os óbitos ocorreram são apresentados na Figura 1 por meio das letras A, B, C, D, E, F, G, H, I, J, K e L. Obteve-se, também, a idade e sexo do trabalhador.

Os dados meteorológicos foram obtidos na reanálise do National Center for Environmental Prediction/National Center for Atmospheric Research (NCEP/NCAR) 21. Esses dados são organizados em pontos de grade uniformemente espaçados a cada $2,5^{\circ}$ de latitude e longitude. Pontos de grade são posições georreferenciadas, para os quais são atribuídos valores de variáveis atmosféricas. As localizações dos pontos de grade utilizados neste estudo são apresentadas na Figura 1 por $\mathrm{P} 1, \mathrm{P} 2, \mathrm{P} 3, \mathrm{P} 4, \mathrm{P} 5, \mathrm{P} 6, \mathrm{P} 7, \mathrm{P} 8, \mathrm{P} 10$ e P11. Os dados do NCEP/NCAR são disponibilizados em quatro horários diários, 00, 06, 12 e 18 UTC. A sigla UTC significa universal time coordinated, referente a um horário padrão internacional adiantado em três horas em relação ao horário oficial de Brasília. A reanálise do NCEP/NCAR é obtida usando-se os dados meteorológicos de superfície em terra, navios, radiossondas, aeronaves, satélites e outros meios, sendo que o controle de qualidade e assimilação desses dados é realizado pelo mesmo sistema desde 1957. Entende-se por assimilação o método utilizado para atribuir a cada ponto de grade o valor das variáveis meteorológicas medidas pelas diferentes formas de observação. Portanto, tanto para os dias em que ocorreram os óbitos como para o período utilizado para obtenção dos parâmetros estatísticos, o conjunto de informações meteorológicas do NCEP/NCAR foi obtido com padrão único de coleta e assimilação de dados. A reanálise do NCEP/NCAR é acessada pelo endereço eletrôni-

Mapa do Estado de São Paulo, Brasil, com a localização das cidades: (A) Valparaíso, (B) Rio das Pedras, (C) Guariba,

(D) Teodoro Sampaio, (E) Jaborandi, (F) Monte Alto, (G) Taiaçu, (H) Itapira, (I) Colina, (J) Ribeirão Preto, (K) Ipaussu e

(L) Presidente Prudente.

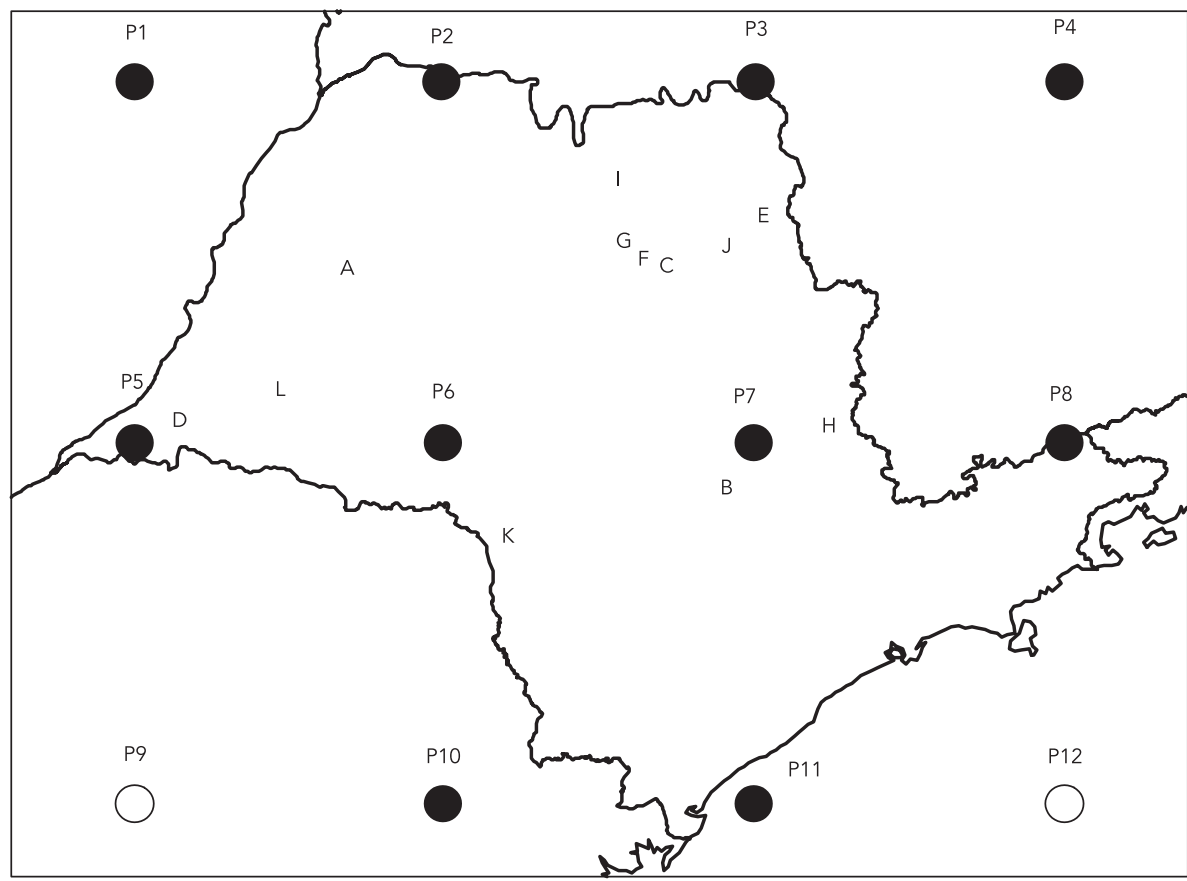

Nota: os círculos fechados mostram a localização dos pontos de grade (P1, P2, P3, P4, P5, P6, P7, P8, P10 e P11), dos quais são obtidas as variáveis meteorológicas. 
co: http://www.cdc.noaa.gov/data/re-analysis / reanalysis.shtml (acessado em 17/Mar/2009).

As variáveis meteorológicas, para os locais onde os óbitos ocorreram, foram obtidas usandose a interpolação dos valores dos pontos de grade localizados ao redor destes locais. Por exemplo, de acordo com a disposição espacial das cidades e dos pontos de grade mostrados na Figura 1, as interpolações para as cidades A, D e L são obtidas com base nos valores dos pontos de grade $\mathrm{P} 1, \mathrm{P} 2$, P5 e P6. O cálculo para interpolação é dado pela seguinte expressão:

$$
\begin{aligned}
V_{L o c}= & \left(\frac{1 / d 1}{1 / d 1+1 / d 2+1 / d 3+1 / d 4}\right) V_{1}+ \\
& \left(\frac{1 / d 2}{1 / d 1+1 / d 2+1 / d 3+1 / d 4}\right) V_{2}+ \\
& \left(\frac{1 / d 1+1 / d 2+1 / d 3+1 / d 4}{1 / d 3}\right) V_{3}+ \\
& \left(\frac{1 / d 4}{1 / d 1+1 / d 2+1 / d 3+1 / d 4}\right) V_{4}
\end{aligned}
$$

em que $V_{L o c}$ é o valor da variável meteorológica no local do óbito, $V_{1}, V_{2}, V_{3}$ e $V_{4}$ são os valores das variáveis meteorológicas nos quatro pontos de grade ao redor e $d 1, d 2, d 3$ e $d 4$ são as distâncias entre estes pontos de grade e o local do óbito. As variáveis meteorológicas analisadas são: temperatura do ar $(T)$, umidade relativa do ar $(U R)$, velocidade do vento $(V)$ e a radiação incidente $(R)$, dada pela soma da radiação de ondas curta e longa, ambas na superfície terrestre. A $T, U R$ e $V$ são da coordenada vertical sigma, que é um nível atmosférico comumente utilizado em modelos numéricos. Esse nível fica próximo da superfície e caracteriza-se por contornar o relevo. As variáveis $T, U R$ e $V$ foram analisadas para o horário das 18 UTC, 15h local. A variável $R$ representa a incidência média que ocorreu na superfície terrestre entre as 12 e 18 UTC, ou seja, entre as 9h e 15h local. A escolha dessas variáveis meteorológicas se deu por serem os parâmetros ambientais medidos em áreas abertas mais importantes para a estimativa de sobrecarga térmica, por meio do Índice de Bulbo Úmido - Termômetro de Globo (IBUTG) 22. A norma ISO 724323 descreve o IBUTG como metodologia de avaliação de estresse térmico do trabalhador, e a Norma Regulamentadora de Segurança e Higiene do Trabalho (NR 15) 24 estabelece-o legalmente como índice térmico brasileiro para avaliação das condições de trabalho em ambientes sob temperaturas elevadas.
Inicialmente, as variáveis $T, U R, V$ e $R$ foram obtidas para o dia e local do óbito e comparadas com a média, desvio-padrão e valores mínimo e máximo, extraídos da série histórica de 1957 a 2008, considerando o mesmo local, dia e mês em que o óbito ocorreu. Não foram feitas comparações estatísticas entre os dados de óbito e os dados meteorológicos devido às significativas diferenças de escala e níveis organizativos existentes entre estas duas bases de dados. Posteriormente, calculou-se a distribuição de frequência da $T$, $U R, V$ e $R$ para todos os dias do ano, no período de 1957 a 2008. Nesse caso, os locais onde ocorreram os óbitos foram representados pela média dos quatro pontos de grade localizados ao redor.

\section{Resultados e discussões}

\section{As condições de trabalho no canavial}

Fischer et al. 9 desenvolveram uma reflexão sobre a organização do trabalho na agroindústria canavieira de São Paulo, e dividiram o processo na fase de obtenção da matéria-prima e na do seu processamento industrial. Os autores subdividem a obtenção da matéria-prima em três principais atividades agrícolas: o plantio, a carpa e o corte.

A análise que se faz neste estudo tem o objetivo de abordar rapidamente as condições de trabalho na fase de obtenção da matéria-prima, em geral, sem distinção de atividades, apesar da maioria dos artigos referenciados tratarem da atividade de corte 1,3,7,8. Geralmente, as condições de trabalho são inadequadas e podem ser resumidas como segue:

(i) O pagamento é feito por produção, o que implica maior dispêndio de energia e de esforços físicos para cortar mais cana 8,25 . A exigência de intensificação da produção decorre da maior demanda da agroindústria do álcool e açúcar ${ }^{9}$;

(ii) Ocorre aquecimento da terra pela prática da queima da cana antes de seu corte. Algumas vezes, o calor se conserva até o início da próxima jornada, contribuindo para a sobrecarga térmica dos trabalhadores 7 . Além disso, a fuligem e a fumaça provocam problemas respiratórios 5;

(iii) A alimentação é insuficiente, inadequada e realizada sem as condições mínimas de higiene. O intervalo de tempo entre a alimentação e o retorno ao trabalho é muito pequeno. Há consumo insuficiente de líquidos e ausência de pausas para descanso durante a jornada de trabalho;

(iv) Os alojamentos, os meios de transporte e as instalações sanitárias sãos precários;

(v) O uso de EPI é improvisado e inadequado à proteção. As vestimentas utilizadas cobrem todo 
o corpo e dificultam a troca de calor necessária ao equilíbrio térmico. Não há equipamentos de primeiros socorros no local de trabalho.

Entre os óbitos registrados pela Pastoral do Migrante de Guariba, poucos possuem informações sobre a causa da morte (Tabela 1). Oficialmente, em nenhum dos casos foi mencionada a ligação com o trabalho excessivo ou calor, apesar dos relatos de familiares e colegas de trabalho indicarem claramente uma dessas relações. Grégoire et al. 17 ao analisarem o efeito de seis ondas de calor na França, identificaram as doenças cardiovasculares e do sistema respiratório como as principais causas para as mortes ocorridas durante os dias quentes. Neste estudo, percebemos que dos cinco casos em que há registro para a causa do óbito, pelo menos três (casos 03, 09 e 14 - Tabela 1) estão diretamente relacionadas com essas doenças. É bastante provável que a maioria dos trabalhadores do corte de cana-de-açúcar, por serem desprovidos de assistência médica adequada, apresente pré-disposição para doenças respiratórias e cardiovasculares e, quando expostos ao estresse térmico e outros fatores prejudiciais à saúde, como a fumaça das queimadas, por exemplo, potencializam a manifestação destas doenças.
Outro fator que merece ser considerado na análise dessas mortes é relativo à idade: 10 dos 14 trabalhadores mortos tinham mais de 35 anos. No estudo de Grégoire et al. 17 foi encontrado que entre $20 \%$ e $33 \%$ das mortes ocorridas durante as ondas de calor da França foram de pessoas com a faixa etária de 35 a 74 anos.

\section{Relação das variáveis meteorológicas com os óbitos}

A ciência que analisa os efeitos das condições atmosféricas sobre a saúde e bem-estar humano é conhecida como biometeorologia 26. É senso comum e também demonstrado por meio de pesquisas que reações adversas sobre a saúde podem ser provocadas por essas condições 25,27. Segundo Tromp 28, os parâmetros meteorológicos que mais afetam as pessoas são: temperatura do ar, umidade do ar, velocidade do vento e radiação, embora a condição extrema de calor ou frio tenha se mostrado de maior significância em termos de morbidade e mortalidade humana 29.

Os óbitos dos cortadores de cana ocorreram em épocas distintas do ano, três nos meses de primavera, seis no outono e cinco no inverno (Tabela 1). Não há registro de mortes nos meses

Óbitos dos cortadores de cana-de-açúcar registrados no Estado de São Paulo, Brasil.

\begin{tabular}{|c|c|c|c|c|c|}
\hline Caso & Data & Cidade & Idade & Sexo & Causa do óbito \\
\hline 01 & 04/Out/2005 & Valparaíso & 43 & M & Desconhecida \\
\hline \multirow[t]{2}{*}{02} & 21/Out/2005 & Rio das & 45 & $M$ & Desconhecida \\
\hline & & Pedras & & & \\
\hline \multirow[t]{2}{*}{03} & 23/Nov/2005 & Guariba & 55 & $\mathrm{M}$ & Edema hemorrágico pulmonar e cardiopatia \\
\hline & & & & & dilatada descompensada \\
\hline \multirow[t]{2}{*}{04} & 13/Abr/2006 & Teodoro & 42 & $\mathrm{~F}$ & Desconhecida \\
\hline & & Sampaio & & & \\
\hline 05 & 29/Jun/2006 & Jaborandi & 37 & M & Desconhecida \\
\hline 06 & 24/Jul/2006 & Monte Alto & 54 & $\mathrm{~F}$ & Desconhecida \\
\hline 07 & 26/Jul/2006 & Taiaçu & 41 & M & Desconhecida \\
\hline 08 & 15/Set/2006 & Itapira & 48 & M & Desconhecida \\
\hline 09 & 28/Mar/2007 & Guariba & 51 & $\mathrm{M}$ & Enfarto do miocárdio \\
\hline 10 & 24/Abr/2007 & Colina & 20 & M & Desconhecida \\
\hline \multirow[t]{2}{*}{11} & 19/Mai/2007 & Ribeirão & 34 & M & Choque anafilático causado por infecção \\
\hline & & Preto & & & \\
\hline 12 & 20/Jun/2007 & Ipaussu & 33 & $\mathrm{M}$ & Desconhecida \\
\hline 13 & 11/Set/2007 & Guariba & 28 & M & Púrpura trombocitopênica idiopática \\
\hline \multirow[t]{2}{*}{14} & 19/Mai/2008 & Presidente & 53 & $\mathrm{M}$ & Parada cardiorrespiratória \\
\hline & & Prudente & & & \\
\hline
\end{tabular}

F: feminino; M: masculino.

Fonte: Pastoral do Migrante 20. 
de verão, pois nesta época normalmente não é feita a colheita da cana-de-açúcar 30 .

Seis dos 14 casos de óbito ocorreram em dias em que a $T$ das $15 \mathrm{~h}$ foi igual ou muito próxima à maior $T$ registrada no mesmo horário e dia durante o período de 1957 a 2008. Isso ocorreu nos casos 01, 06, 07, 08, 09 e 12 (Figura 2a). Os casos 02, 04, 10 e 14 apresentaram $T$ superior à média climatológica distando desta, pelo menos, um desvio-padrão, indicando uma condição de muito calor para o dia em questão. Os casos 03, 11 e 13 tiveram $T$ acima da média, mas dentro do intervalo de um desvio-padrão. Somente o caso 05 apresentou $T$ abaixo da média, coincidindo com o limite inferior do desvio-padrão. A metade dos óbitos ocorreu em dias com $T$ superior a $27^{\circ} \mathrm{C}$ e, conforme análise da Figura 3a, os valores iguais ou superiores a $27^{\circ} \mathrm{C}$ ocorrem numa frequência média de 16,4\%, dependendo da localização da cidade.

A baixa umidade relativa possibilita a perda de calor por evaporação, contribuindo para diminuição da sobrecarga térmica, mas as vestimentas utilizadas pelos cortadores de cana prejudicam significativamente este processo. Em três casos com $T$ igual ao valor máximo histórico, registrou-se $U R$ mínimas históricas (Figura 2b). A comparação dos gráficos apresentados nas Figuras $2 \mathrm{a}$ e $2 \mathrm{~b}$ também mostra que, na maioria das vezes, quando a $T$ fica acima da média climatológica, a UR fica abaixo da média. Há apenas três exceções. Nos casos 10 e 14 a $T$ apresentou valor acima da média e a $U R$ valor igual ou muito próximo da média. No caso 11 , a $T$ e a $U R$ ficaram pouco acima da média climatológica. Isso indica que embora o caso 11 não tenha sido de $T$ muito alta $\left(T=25,2^{\circ} \mathrm{C}\right.$ às $\left.15 \mathrm{~h}\right)$, a umidade mais elevada que a normal pode ter contribuído para maior sobrecarga térmica.

$\mathrm{O}$ vento pode minimizar os efeitos do calor sobre os trabalhadores da cana-de-açúcar. Entretanto, na maioria dos casos de óbitos, 12 casos, a velocidade do vento ficou abaixo da média climatológica. Nos dois casos restantes o valor da velocidade do vento ficou dentro dos limites de desvio-padrão. Em nenhum caso de óbito a velocidade do vento ultrapassou o valor de $5 \mathrm{~m} . \mathrm{s}^{-1}$ (Figura 2c).

A soma das radiações de onda curta e de onda longa incidente sobre os trabalhadores da canade-açúcar é outro parâmetro importante para a qualificação da sobrecarga térmica. Nos casos 06 , 07, 08, 12 e 13, a radiação no dia do óbito atingiu ou ficou muito próxima do valor máximo da série histórica (Figura 2d). Nos quatro primeiros casos, o mesmo aconteceu em relação a $T$ (Figura 2a). Em três casos, a radiação observada no dia do óbito foi igual ou muito próxima da média climatológica. E, nos cinco casos restantes, ficou abaixo da média climatológica, sendo que no caso 05 o valor de $R$ foi igual ao recorde inferior de toda a série histórica, coincidindo com o caso de menor temperatura.

\section{Distribuição de frequência das variáveis meteorológicas no período de 1957 a 2008}

O oeste do Estado de São Paulo, onde se localizam as cidades de Valparaíso, Teodoro Sampaio e Presidente Prudente (Região 1), apresenta as ocorrências de $T$ mais elevadas (Figura 3a). Nessa região, valores iguais ou superiores a $27^{\circ} \mathrm{C}$ ocorreram em cerca de $28,3 \%$, enquanto na região de Guariba, Monte Alto, Taiaçu, Colina e Ribeirão Preto (Região 2) a frequência foi de $18,8 \%$. Para as outras duas regiões, a ocorrência de $T$ igual ou superior a $27^{\circ} \mathrm{C}$ é de $9,7 \%$ nos municípios de Jaborandi e Itapira (Região 3) e de 8,8\% nos municípios de Rio das Pedras e Ipaussu (Região 4). Especificamente para o período que se tem registro de óbitos nos canaviais de São Paulo (outubro de 2005 - maio de 2008) a ocorrência de dias com $T$ igual ou superior a $27^{\circ} \mathrm{C}$ é maior, $37,9 \%$ na Região $1,24,8 \%$ na 2 , $14,7 \%$ na 3 e 13,4\% na Região 4 (dados não mostrados em figura).

Valores de $T$ acima de $30^{\circ} \mathrm{C}$ ocorrem em $11,2 \%, 7,4 \%, 2,7 \%$ e $2,2 \%$, respectivamente, nas regiões 1,2 , 3 e 4 . Valores acima de $33^{\circ} \mathrm{C}$, considerados como muito altos, possuem frequência de aproximadamente $3,8 \%$ e 1,6\%, respectivamente, nas regiões 1 e 2 , e de aproximadamente $0,2 \%$ nas regiões 3 e 4 .

No período estudado, 51 anos, os dias de maior umidade relativa (entre $80 \%$ e $100 \%$ ) ocorreram com maior frequência em todas as cidades onde foram registrados os óbitos (Figura 3b). Entretanto, devido a $U R$ tender a ser maior quanto menor for a $T$, não se pode afirmar que os dias com alta umidade são coincidentes com os dias de elevadas temperaturas.

Por outro lado, em cerca de $50 \%$ dos dias observaram-se ventos significativos, entre 2 e 4 m.s $\mathrm{s}^{-1}$, que favoreceram as trocas térmicas (Figura 3c), embora o seu efeito seja minimizado devido a barreira estabelecida pela vestimenta usualmente utilizada. Velocidades acima de $8 \mathrm{~m} . \mathrm{s}^{-1}$ ocorreram em aproximadamente $1 \%$ dos dias. Em Rio das Pedras e Ipaussu a ocorrência de velocidades do ar superiores a 4 m.s-1 foi maior que nas outras cidades onde se registraram morte de trabalhadores.

A soma da radiação de ondas curta e longa apresentou valores entre 950 e 1150 W.m-2, que corresponde ao intervalo entre $31 \%$ e $37 \%$ dos dias analisados (Figura 3d). 
Relação entre os valores das variáveis meteorológicas quando da ocorrência das mortes com os da série histórica.

2a) Temperatura do $\operatorname{ar}\left({ }^{\circ} \mathrm{C}\right)$

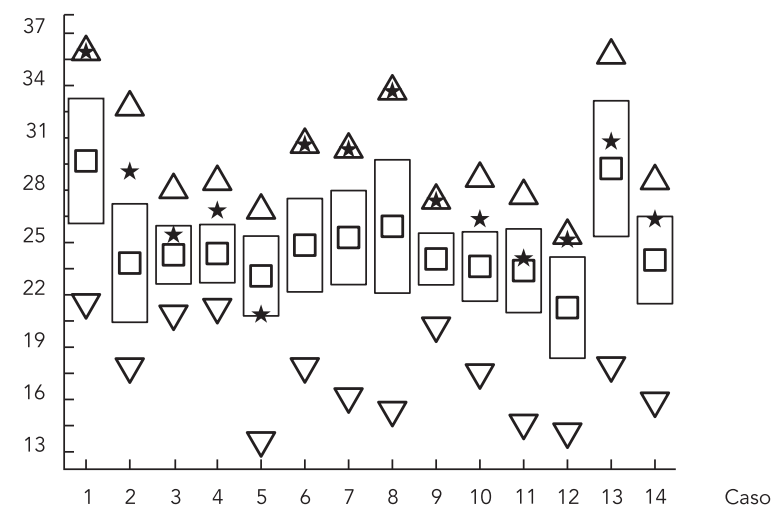

2c) Velocidade do vento $\left(m . s^{-1}\right)$

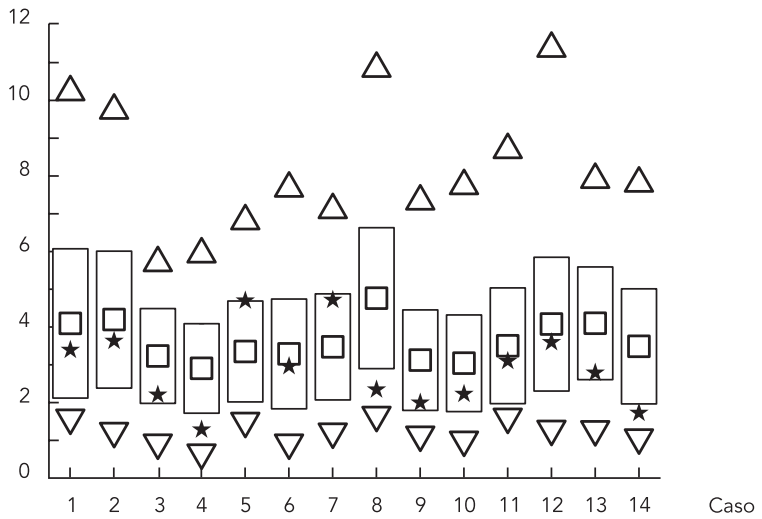

2b) Umidade relativa do ar (\%)

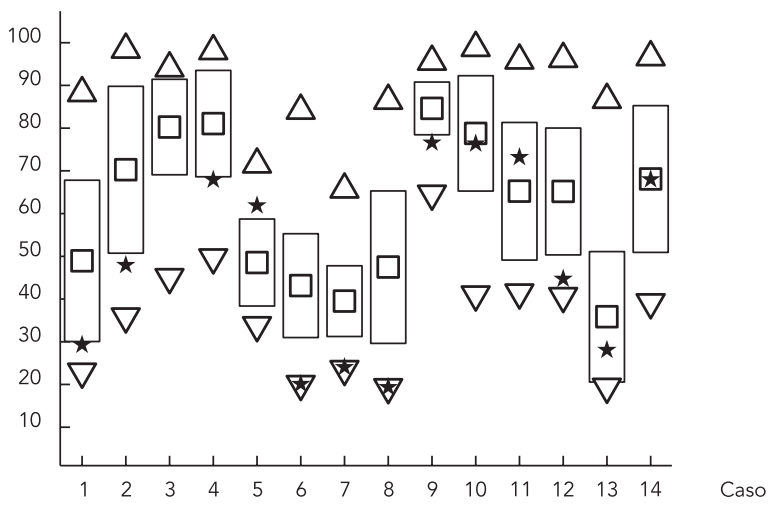

2d) Radiação (W.m-2)

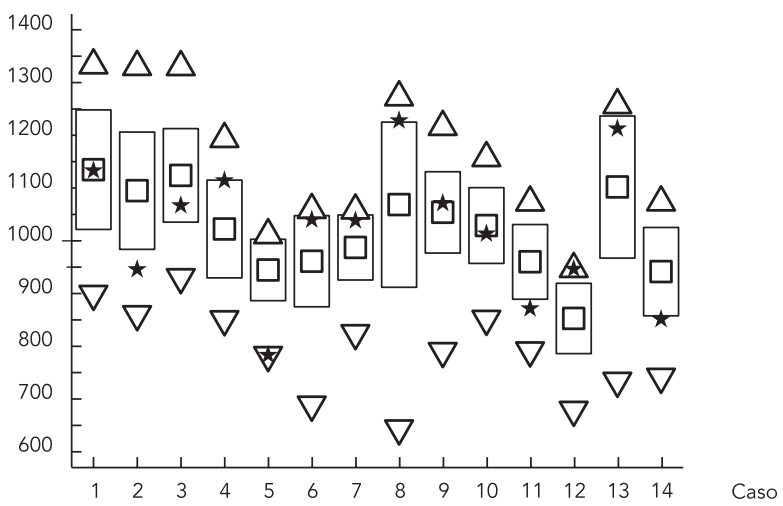

Nota: o símbolo $\star$ mostra: (a) $T$, (b) UR, (c) Ve (d) $R$ no dia e local de ocorrência do óbito. Para cada caso, os gráficos mostram a média climatológica ( $\square$ ), o desvio-padrão acima e abaixo da média (caixa maior) e os valores mínimo ( $\nabla)$ e máximo ( $\triangle$ ) extraídos da série histórica de 1957-2008. Os casos de óbitos são referentes aos registros apresentados na Tabela 1.

Percebe-se por meio dos gráficos da Figura 3 que os valores dos parâmetros que podem levar esses trabalhadores à sobrecarga térmica são relativamente comuns nas cidades que registraram os óbitos. Esses resultados são coerentes com as características climáticas do Estado de São Paulo e, por isto, podem ser válidos para a maioria das cidades paulistas.

\section{Conclusões}

Os artigos que abordam esse tema apontam o excessivo esforço físico na atividade de corte de cana, incentivado pelo ganho por produção, como a principal causa dos óbitos. Os estudos anteriores mostram as más condições de trabalho, mencionando rapidamente o calor na região de São Paulo como fator contribuinte. Por isso, a principal contribuição deste artigo é a discussão sobre a possível contribuição das variáveis meteorológicas, utilizadas na estimativa da sobrecarga térmica, para a ocorrência dos 14 casos 
Distribuição de frequência para intervalos fixos de (a) T, (b) UR, (c) V e (d) R. Os valores são representativos das cidades onde os óbitos ocorreram, conforme legenda.

3a) Temperatura do ar ( $\left.{ }^{\circ} \mathrm{C}\right)$

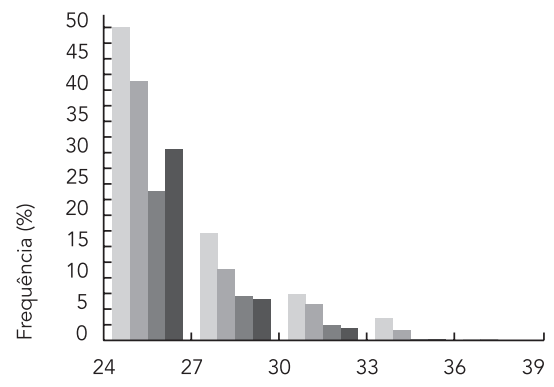

$$
\begin{aligned}
& \text { [ Valparaiso, Teodoro Sampaio e Presidente Prudente } \\
& \text { [ Guariba, Monte Alto, Taiaçú, Colina e Ribeirão Preto } \\
& \text { Jaborandí e Itapira } \\
& \text { Rio das Pedras e Ipaussu }
\end{aligned}
$$

3b) Umidade relativa do ar (\%)

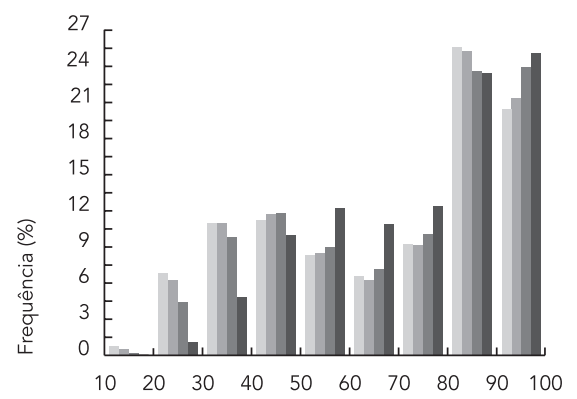

[ Valparaiso, Teodoro Sampaio e Presidente Prudente Guariba, Monte Alto, Taiaçú, Colina e Ribeirão Preto Jaborandí e Itapira

Rio das Pedras e Ipaussu

3c) Velocidade do vento (m.s-1)

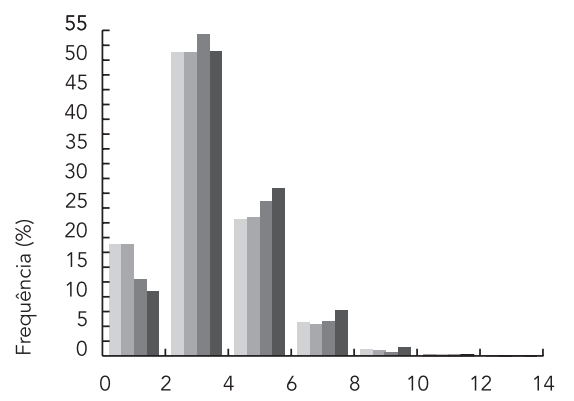

[ Valparaiso, Teodoro Sampaio e Presidente Prudente Guariba, Monte Alto, Taiaçú, Colina e Ribeirão Preto Jaborandí e Itapira

Rio das Pedras e Ipaussu

3d) Radiação (W.m-2)

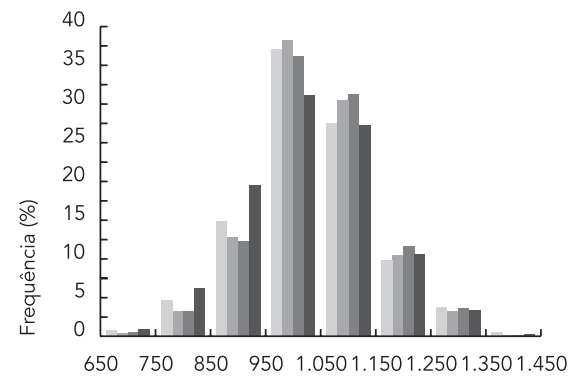

| Valparaiso, Teodoro Sampaio e Presidente Prudente | Guariba, Monte Alto, Taiaçú, Colina e Ribeirão Preto Jaborandí e Itapira

Rio das Pedras e Ipaussu 
de óbitos de cortadores de cana-de-açúcar. Os valores dessas variáveis, obtidos para os dias dos óbitos, foram comparados com os parâmetros estatísticos da série histórica de 1957 a 2008.

O conjunto de informações disponíveis indica que a maioria das mortes possivelmente tenha ocorrido devido a combinação da precariedade da saúde individual do trabalhador, más condições de trabalho, excesso de esforço físico e sobrecarga térmica. Apesar da temperatura ter atingido valores iguais ou muito próximos do recorde histórico em seis dos 14 casos de óbitos, as precárias condições sociais, econômicas e de trabalho desses trabalhadores não permitem apontar o fator atmosférico como causa predominante para os óbitos. Entretanto, é possível concluir que a condição meteorológica pode ser um fator importante a ser considerado na análise das condições gerais de trabalho desses trabalhadores.

Neste estudo, foram utilizados dados meteorológicos obtidos da reanálise do NCEP/NCAR que, embora demonstrem adequadamente a ocorrência de ondas de calor, não são dados observados exatamente nos canaviais onde os óbitos ocorreram. Além disso, há grande carência de detalhamento para os dados de óbitos, principalmente no que diz respeito à causa das mortes e à falta de informação do contingente de trabalha- dores. Essas características somadas à significativa diferença de escala e níveis organizativos entre essas duas bases de dados são fatores limitantes para as constatações deste estudo. Sugere-se, portanto, o desenvolvimento de estudos específicos sobre as causas de óbitos como indicadores das condições de trabalho dessa população de trabalhadores, nos quais as condições atmosféricas sejam também consideradas.

Também sugere-se estudos que realizem o monitoramento das variáveis atmosféricas em paralelo à vigilância das condições de trabalho dos trabalhadores rurais. Considera-se importante repensar a organização do trabalho de maneira a diminuir a sua sobrecarga térmica, adequando o seu ritmo de trabalho às condições atmosféricas. Nesse sentido, o aumento da frequência de pausas para descanso é uma medida de proteção importante, principalmente nos dias mais quentes. Essa medida e outras como o estímulo à hidratação, a ingestão controlada de sais, a aclimatização e o monitoramento do calor por meio de avaliações ambientais nos mesmos locais e hora onde o trabalho é realizado deveriam ser aplicadas até que novos estudos desenvolvam um limite de segurança relacionado ao conjunto de parâmetros meteorológicos para o trabalho a céu aberto em dias quentes.

\section{Resumo}

Os cortadores de cana-de-açúcar exercem atividade muito pesada e em condições precárias de trabalho. Além disso, fatores ambientais podem ser um importante agravante à sobrecarga térmica desses trabalhadores. Neste artigo, analisa-se a condição atmosférica no dia da morte de 14 trabalhadores do corte de canade-açúcar no Estado de São Paulo, Brasil. Compara-se valores estatísticos históricos da temperatura, umidade, vento e radiação com os valores destas variáveis meteorológicas observadas no dia de cada óbito. Os dados atmosféricos foram obtidos da reanálise do $\mathrm{Na}$ tional Center for Environmental Prediction/National Center for Atmospheric Research (NCEP/NCAR). Em 10 dos 14 casos analisados, as temperaturas observadas nos dias dos óbitos foram maiores ou iguais à média somada ao desvio-padrão. Em seis desses casos o valor da temperatura atingiu ou ficou muito próxima do recorde histórico. Constatou-se que a condição atmosférica pode ser um fator importante a ser considerado na análise das condições gerais de trabalho dos cortadores de cana-de-açúcar.

Transtorno de Estresse por Calor; Saúde do Trabalhador; Atmosfera

\section{Colaboradores}

D. P. Bitencourt participou na concepção e projeto, análise e interpretação dos dados, redação do artigo e aprovação final da versão a ser publicada. A. C. Ruas e P. A. Maia colaboraram na concepção e projeto, análise e interpretação dos dados, revisão crítica relevante do conteúdo intelectual e aprovação final da versão a ser publicada.

\section{Agradecimentos}

Agradecemos à Pastoral do Migrante de Guariba pelos registros de óbitos e ao National Center for Environmental Prediction/National Center for Atmospheric Research (NCEP/NCAR) pelos dados meteorológicos. Agradecemos aos colaboradores Cloduardo Gomes da Silva, Luiz Roberto Monteiro e Marcelo Cândido de Oliveira pelas discussões acerca do tema estudado e contribuições obtidas em pesquisa de campo. 


\section{Referências}

1. Scopinho RA, Eid F, Vian CEF, Silva PRC. Novas tecnologias e saúde do trabalhador: a mecanização do corte da cana-de-açúcar. Cad Saúde Pública 1999; 15:147-61.

2. Petti R, Fredo CE. Emprego formal na cana-deaçúcar. ftp://ftp.sp.gov.br/ftpiea/AIA/AIA-012009. pdf (acessado em 08/Set/2009).

3. Juttel LP. Esforço físico excessivo busca aumento de renda. Ciênc Cult (São Paulo) 2008; 60:6-7.

4. Tatsch JD. Uma análise dos fluxos de superfície e do microclima sobre cerrado, cana-de-açúcar e eucalipto, com implicações para mudanças climáticas regionais [Dissertação de Mestrado]. São Paulo: Universidade de São Paulo; 2006.

5. Ribeiro H. Queimadas de cana-de-açúcar no Brasil: efeitos à saúde respiratória. Rev Saúde Pública 2008; 42:370-6.

6. Cançado JED. A poluição atmosférica e sua relação com a saúde humana na região canavieira de Piracicaba - SP [Tese de Doutorado]. São Paulo: Universidade de São Paulo; 2003.

7. Alessi NP, Navarro VL. Saúde e trabalho rural: o caso dos trabalhadores da cultura canavieira na região de Ribeirão Preto, São Paulo, Brasil. Cad Saúde Pública 1997; 13 Suppl 2:111-21.

8. Alves F. Por que morrem os cortadores de cana? Saúde Soc 2006; 15:90-8.

9 Fischer A, Fleury MTL, Ferreira RMF. O processo e organização do trabalho na agroindústria canavieira. Revista de Administração 1984; 19:17-22.

10. Silva MAM. Trabalho e trabalhadores na região do "mar de cana e do rio do álcool". Agrária 2005; (2):2-39.

11. Basu R, Samet JM. Relation between elevated ambient temperature and mortality: a review of the epidemiologic evidence. Epidemiol Rev 2002; 24:190-202.

12. Gasparrini A, Armstrong B. The impact of heat waves on mortality. Epidemiology 2011; 22:68-73.

13. Sheridan S, Kalkstein L, Kalkstein A, Greene S. Heat-related mortality and heat watch-warning systems in the United States: recent developments. Epidemiology 2011; 22 Suppl:S13.

14. Lee D-G, Choi Y-J, Kim KR, Kalkstein L, Sheridan S. Regional characteristics of heat-related deaths and the application of a heat-health warning system in Korea. Epidemiology 2011; 22 Suppl:S180.

15. Chung JY, Kim H, Honda Y. Ambient temperature and mortality: an international study in 13 cities of East Asia. Epidemiology 2011; 22 Suppl:S21.

16. Pascal M, Wagner V, Le Tertre A, Laaidi K, Beaudeau, P. Choice of temperature thresholds for the French heat warning system. Epidemiology 2011; 22 Suppl:S14.
17. Grégoire R, Jougla E, Fouillet A, Pavillon G, Bessemoulin P, Frayssinet P, et al. The impact of major heat waves on all-cause and cause-specific mortality in France from 1971 to 2003. Int Arch Occup Environ Health 2007; 80:615-26.

18. Dhainaut J-F, Claessens Y-E, Ginsburg C, Riou B. Unprecedented heat-related deaths during the 2003 heat wave in Paris: consequences on emergency departments. Crit Care 2004; 8:1-2.

19. Milojevic A, Wilkinson P, Armstrong B, Davis M, Mavrogianni A, Bohnenstengel S, et al. Impact of London's urban heat island on heat-related mortality. Epidemiology 2011; 22 Suppl:S182-3.

20. Pastoral do Migrante. Histórico dos cortadores de cana mortos no setor canavieiro. http://www.pas toraldomigrante.com.br/index.php?option $=\mathrm{com}_{-}$ content\&view $=$ article\&id $=102 \&$ Itemid $=60$ (acessado em 20/Out/2009).

21. Kalnay E, Kanamitsu M, Kistler R, Collins W, Deaven D, Gandin L, et al. The NCEP/NCAR 40-year reanalysis project. Bulletin of the American Meteorological Society 1996; 77:437-71.

22. Hunter $\mathrm{CH}$, Minyard CO. Estimating wet bulb globe temperature using standard meteorological measurements. In: 2nd Symposium on Environmental Applications. Boston: American Meteorological Society; 2000. p. 9-14.

23. International Organization Standardization. ISO 7243: hot environments: estimation of the heat stress on working man. Geneva: International Organization Standardization; 1989.

24. Ministério do Trabalho. Portaria no ${ }^{\circ} .214$, de 08 de junho de 1978. Normas Regulamentadoras: NR-15 - atividades e operações insalubres. In: Ministério do Trabalho, organizador. Manual de legislação: atlas de segurança e medicina do trabalho. 62a Ed. São Paulo: Editora Atlas; 2008. p. 138-220.

25. Bart JL, Bourque DA. Acknowledging the weatherhealth link. CMAJ 1995; 153:941-4.

26. Driscoll DM. Human health. In: Houghton DD, editor. Handbook of applied meteorology. New York: Wiley; 1985. p. 778-814.

27. Derrick EH. The seasonal variation of asthma in Brisbane: Its relation to temperature and humidity. Int J Biometeorol 1965; 9:239-51.

28. Tromp SW. Biometeorology. London: Heyden; 1980.

29. McMichael AJ, Haines A, Sloof R, Kovats S. Climate change and human health. Geneva: World Health Organization; 1996.

30. Agrobyte. Cana-de-açúcar (Saccharum hibridas). http://www.agrobyte.com.br/index.php?pag= cana (acessado em 27/Out/2009).

Recebido em 30/Jun/2010

Versão final reapresenta em 05/Ago/2011 Aprovado em 22/Ago/2011 